\title{
Photoluminescence characterization of few-nanocrystals electronic devices
}

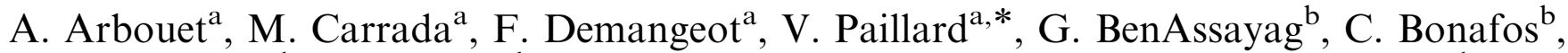

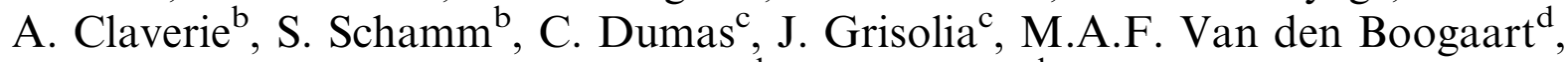 \\ J. Brugger ${ }^{\mathrm{d}}$, L. Doeswijk ${ }^{\mathrm{d}}$ \\ ${ }^{a}$ LPST, Université Paul Sabatier, 118 route de Narbonne, 310062 Toulouse, France \\ ${ }^{\mathrm{b}}$ CEMES-CNRS, 29 rue Jeanne Marvig, 31055 Toulouse, France \\ ${ }^{\mathrm{c}}$ LNMO, INSA, 135 avenue de Rangueil, 31077 Toulouse, France \\ ${ }^{\mathrm{d}}$ LMIS1, EPFL, BM3.202, station 17, 1015 Lausanne, Switzerland
}

Available online 2 October 2006

\begin{abstract}
Photoluminescence (PL) spectroscopy is demonstrated as a suitable technique to characterize silicon nanocrystals (Si-NCs)-based nonvolatile memory devices. The $2 \mathrm{D}$ array of Si-NCs forming the floating gate is obtained by low-energy ion implantation in thin oxide followed by annealing. The electrical properties and PL emission are dramatically improved after annealing in oxidizing conditions, which are necessary conditions for oxide healing. Both these elaboration and characterization techniques are currently extended to the production and the study of nanoscale electronic devices, which exploit the Coulomb blockade effect and other quantized charging features at $300 \mathrm{~K}$. The fabrication of such devices requires the fine control of a small number of NCs of about $3 \mathrm{~nm}$ in diameter. This could be achieved by $1 \mathrm{keV} \mathrm{Si-implantation} \mathrm{through} \mathrm{stencil} \mathrm{masks} \mathrm{with} \mathrm{window} \mathrm{sizes} \mathrm{ranging} \mathrm{from} \mathrm{about} 0.1$ to $10 \mu \mathrm{m}^{2}$, with spacing between apertures in the $1-10 \mu \mathrm{m}$ range. After mask removal and annealing, PL spectroscopic imaging under a confocal microscope is used to detect the NCs rich areas. The image of the PL intensity is found to mimic the mask geometry. Some changes of the PL energy at maximum intensity are found, that could be attributed to different Si-NCs sizes.
\end{abstract}

(C) 2006 Elsevier B.V. All rights reserved.

\section{Introduction}

It is planned to use silicon nanocrystals ( $\mathrm{Si}-\mathrm{NCs}$ ) in new devices to benefit of their original electronic and optical properties [1-3]. Technological challenges are not only the control of NCs size and density, but also their spatial arrangement and the number that can be electrically addressed.

Ultra-low energy Si ion implantation (about $1 \mathrm{keV}$ ) in a thin gate oxide $(5-10 \mathrm{~nm})$ was successfully used to produce, after annealing, a single plane of Si-NCs perfectly positioned at a tunneling distance of the $\mathrm{Si}$ wafer [4]. However, first photoluminescence (PL) measurements showed a very low PL intensity and no evidence of any NC size effect.

\footnotetext{
${ }^{*}$ Corresponding author. Tel.: + 33561558630 ; fax: 33561556233 .

E-mail address: vincent.paillard@lpst.ups-tlse.fr (V. Paillard).
}

Recently, it was shown that annealing in a mild oxidizing atmosphere (either at the same time that the NC growth phase or during a second annealing following the initial growth phase) strongly improved both the electrical and optical properties of these systems [5-6]. For instance, a MOS capacitor showed a better retention time of charges in the $\mathrm{Si}-\mathrm{NCs}$ reservoir, and some quantized charging effects [5]. The emission intensity was also dramatically increased, and the PL energy was shifted according to the average Si-NCs size measured by electron microscopy [6]. The benefits were attributed to the separation of adjacent (coalesced) NCs, as well as to the healing of both the injection and control oxides (in other words the oxide layers on each side of the Si-NCs plane). Indeed, the implantation-induced defects in the silica matrix, some of them in the form of an excess of isolated $\mathrm{Si}$ atoms, still remain after standard annealing in a $\mathrm{N}_{2}$ ambient. The role of the oxidizing annealing is to eliminate the excess $\mathrm{Si}$ 
atoms, leaving isolated and passivated $\mathrm{Si}-\mathrm{NCs}$ inside a high barrier of stoechiometric silica.

Thus, PL spectroscopy is very sensitive to both the $\mathrm{Si}-\mathrm{NCs}$ and the silica matrix quality. Previously, we have shown that a near infrared band was present in samples annealed under inert atmosphere, and disappeared progressively after oxidation [6]. Now, since both the production and characterization techniques are quite well understood, it becomes possible to apply them to both the fabrication and control of new devices containing less NCs. This is an important step in the downscaling of CMOS technology.

There are several methods based on electron beam lithography to obtain electrical circuits driving a few units of NCs, or even a single one. However, this complex process has many disadvantages. One of them, not the less important, is the poor reproducibility of electrical performances.

We present here a process using ion implantation in thin silica, through stencil masks formed of a periodic array of apertures having different geometries and sizes (Fig. 1). The first interrogation is about the formation of Si-NCs restricted to the implanted zones after stencil mask removal and adequate annealing (such as described above).

\section{Experiment}

Samples are produced by implantation of $1 \mathrm{keV}$ Si ions in $7 \mathrm{~nm}$-thick silica layers thermally grown on $\left(\begin{array}{lll}1 & 0 & 0\end{array}\right) \mathrm{Si}$ wafers, to a dose of $10^{16} \mathrm{~cm}^{-2}$. The stencil mask is placed directly on the silica surface and maintained by double sided adhesive tape. After implantation, the stencil mask is removed and annealing is performed. All NCs are initially grown in a nitrogen atmosphere for $30 \mathrm{~min}$ at $1050{ }^{\circ} \mathrm{C}$. Some samples are subsequently annealed under a mixture of $\mathrm{N}_{2}+1.5 \% \quad \mathrm{O}_{2}$ for $30 \mathrm{~min}$ at $950{ }^{\circ} \mathrm{C}$. The samples described in this paper and labeled A and B, correspond to the stencil mask ST6 shown in Fig. 1a. Sample A was annealed in $\mathrm{N}_{2}$, and sample $\mathrm{B}$ was annealed and oxidized.

After the implantation process, the damaged zones can be seen under an optical microscope. However, after annealing, nothing can be seen and the surface sample returns identical to the initial wafer. In such conditions, finding a region containing NCs is quite uneasy, except using PL.

PL spectroscopy is performed using a Dilor XY Raman spectrometer equipped with a super notch filter used to cut the $488 \mathrm{~nm}$ excitation laser line, a 150 groves $\mathrm{mm}^{-1}$ grating and a liquid nitrogen-cooled CCD detector. Of course, imaging is performed thanks to an automated $\mathrm{XY}$ table (steps as small as $0.1 \mu \mathrm{m}$ ). The incident and emitted lights are focused and collected using a confocal microscope equipped either with a $100 \times$ magnification objective working in air (0.9 numerical aperture (NA), or with an oil immersion $100 \times$ objective (1.3 NA). The oil droplet gave no fuorescence in our experimental conditions. The confocal hole was closed down to about $150 \mu \mathrm{m}$ to enhance the in-plane spatial resolution while keeping sufficiently intense signal. The laser power at the entrance of the microscope is $150 \mu \mathrm{W}$ or less. Increasing the laser power does not lead to a linear increase of the PL intensity. In contrary, PL tends first to saturate quickly before disappearing.

We estimate the best spatial resolution to about $0.3 \mu \mathrm{m}$ (to be confirmed in future experiments with dense arrays of sub-micron apertures). A typical high-resolution scan (0.1 $\mu \mathrm{m}$ step) over a large area (several microns squared) extended over $10 \mathrm{~h}$. Using both Jobin-Yvon and homemade softwares, we were able to draw maps of two kinds, one corresponding to the intensity variation, and a spectroscopic one showing the energy of the PL band maximum.

We have not yet succeeded in obtaining energy filteredtransmission electron microscopy (EFTEM) images of NCs formed through stencil masks. In fact, it is highly difficult to etch the samples exactly in a region where Si-NCs are present. Work is in progress to detect NCs formed with stencil masks.

In fact, the size distribution in the case of large windows (in the micron range) should not be different from the size distribution measured in the case of "standard samples" (i.e. implantation of the bare wafer without mask). This is due to the very limited diffusion coefficient of silicon in silica (about one order of magnitude smaller than our large
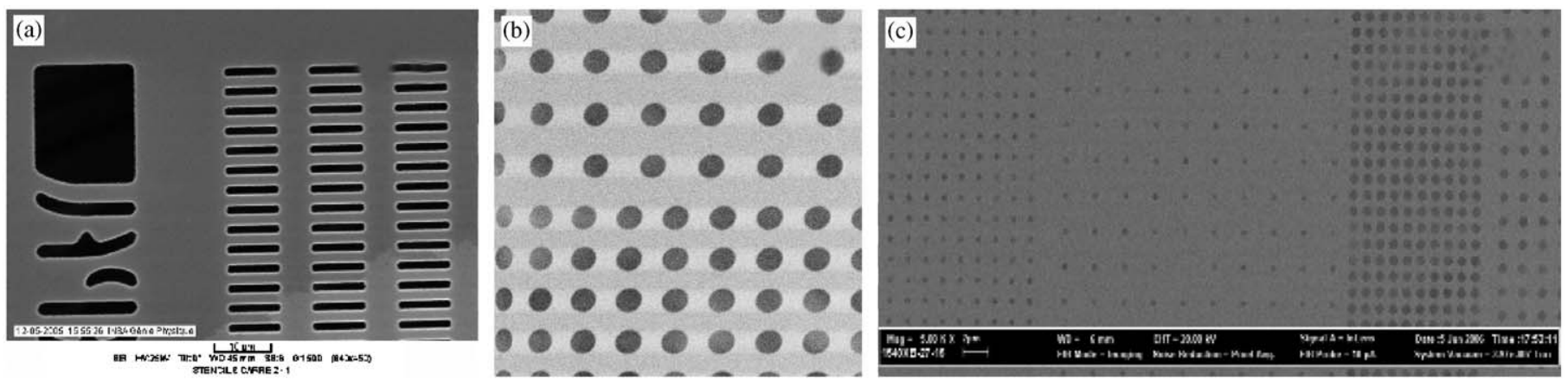

Fig. 1. Optical images of different stencil masks placed on the Si wafers for localized implantation. Aperture shapes as rods of $1 \times 10 \mu \mathrm{m}^{2}$, step of $2.5 \mu \mathrm{m}$ (1a), as disks of $0.9 \mu \mathrm{m}^{2}$ in diameter and steps of about 1.5 and $2.5 \mu \mathrm{m}(1 \mathrm{~b})$, and as sub-micron-scale disks spaced of $200-500 \mathrm{~nm}$ (1c). 
aperture dimensions). Nevertheless, the size is not the important parameter only, the NCs density is also important. From plane-view EFTEM images obtained on

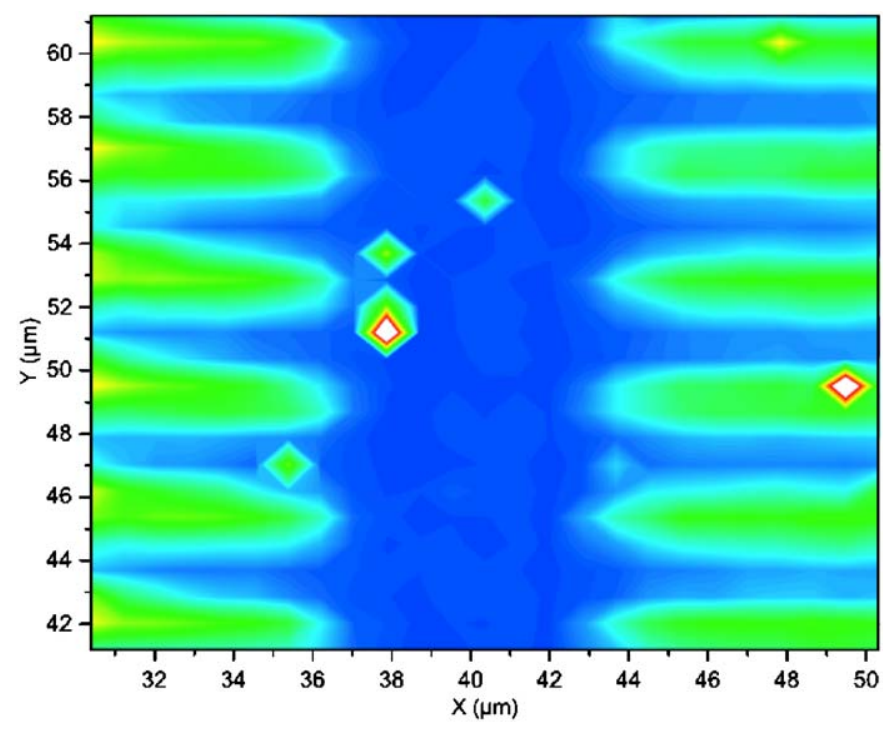

Fig. 2. Large-scale photoluminescence image of localized formation of SiNCs mimicking the stencil mask of Fig. 1a.

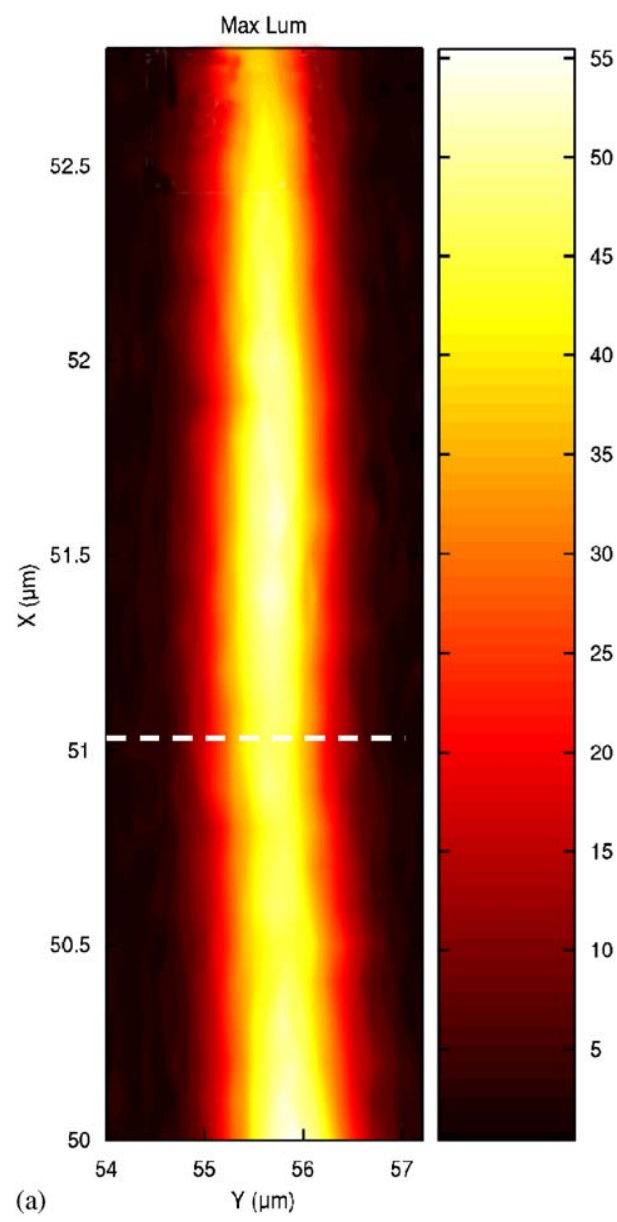

"standard samples", we measured it in the $2 \times 10^{11}$ $2 \times 10^{12}$ particles per $\mathrm{cm}^{2}$ range. This corresponds roughly to $10^{4}-2 \times 10^{4}$ particles under the laser spot of $1 \mu \mathrm{m}$ diameter in a large window and the confocal hole fully opened.

\section{Results and discussion}

A typical large scale PL image taken in large apertures (micron size such as in Fig. 1a) is shown in Fig. 2. It was recorded using the $0.9 \mathrm{NA}$ objective and using a 0.2 micron step. The PL image perfectly fits the stencil mask image. In Fig. 3 are shown images of a zoom. Fig. 3a corresponds to the PL intensity and Fig. $3 b$ to the energy at maximum intensity.

A profile can be obtained by drawing a line along the $Y$-axis (see Fig. 3). The profiles given in Fig. 4 correspond to all PL spectra recorded along the line (at fixed $X$ ordinate). The $Z$-axis gives the PL intensity. We show for comparison the profiles corresponding to sample A (single $\mathrm{N}_{2}$ annealing) and $\mathrm{B}\left(\mathrm{N}_{2}\right.$ annealing followed by oxidizing annealing in $\mathrm{N}_{2} / \mathrm{O}_{2}$ ambient). Sample B spectra are slightly blueshifted compared to sample $\mathrm{A}$, in good agreement with the smaller average size obtained after

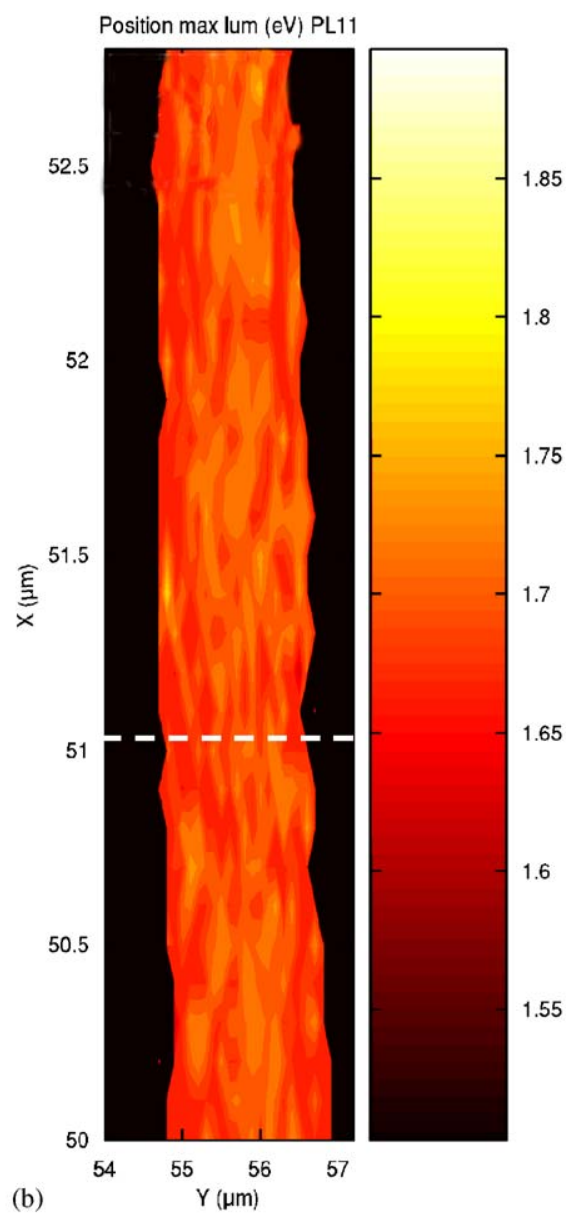

Fig. 3. Zoom on one aperture. The PL intensity and the PL energy at maximum intensity is shown are shown in Figs. 3a and b, respectively. The line (white dashed line) corresponds to the spectral profile given in Fig. 4. 

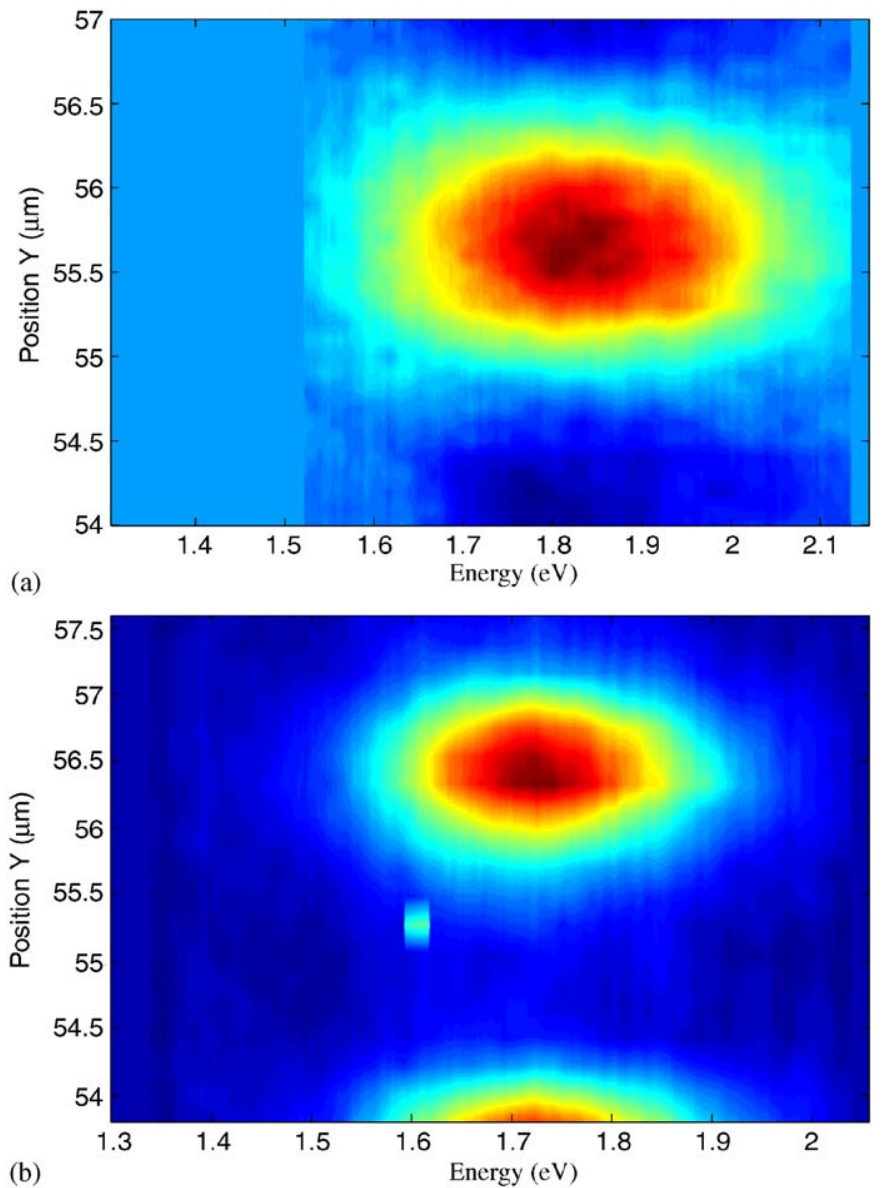

Fig. 4. Profiles corresponding to sample A (inert gas annealing, Fig. 4a) and sample B (oxidized, Fig. 4b) show the shift due a size effect. The profiles are symmetric proving an homogeneous size repartition along the line.

oxidation. As mentioned in the previous section, size measurements using TEM are planned. We can, however, guess that PL maxima located around 1.75 and $1.82 \mathrm{eV}$ should correspond to sizes smaller than $3 \mathrm{~nm}$ in diameter [6-7]. Nevertheless, such samples were used first to validate our production and characterization techniques. According to our previous works, they are both known to give an intense PL. A spectrum is recorded in a few seconds and the presence of NCs in a region can be detected in only $1 \mathrm{~s}$. Though it is not the aim of the preliminary work, we show that the annealing conditions have also a strong influence on the optical properties. The oxidation is in our case a mean to adjust the $\mathrm{NC}$ size independently of the growth phase during $\mathrm{N}_{2}$ annealing, as well as to heal the oxide matrix. Electrical measurements have shown this improve- ment [4], but it must be shown in the present case, with a special attention paid to the reproducibility of such measurements.

There is sometimes an asymmetry of the energy at maximum intensity (Fig. 2b), characterized by a slight color difference from one side of the window to the other, or on both sides compared to the aperture center. This asymmetry is also evidenced in the corresponding profiles with a distorted image compared to those presented in Fig. 3. We thought initially that it was due to a size effect near the aperture edges (less Si implanted atoms than in the window center).

We observed that the asymmetry is not reproducible from one area to another (though it is reproducible in the case of successive scans of the same area). It is certainly due to a NC size effect introduced by a shadowing effect during the implantation phase. We believe that imperfect positioning and/or defects of the stencil mask should affect locally the exact implanted $\mathrm{Si}$ atom concentration.

\section{Conclusion}

As a conclusion, we have a spectroscopic optical technique able to detect localized NCs and sensitive to both their size and environment. Our very last experiments on arrays of holes of $0.9 \mu \mathrm{m}$ in diameter and spaced of the same length are very promising. We are currently investigating the influence of the confocal hole to optimize the spatial resolution versus the acquisition time.

Further experiments are planned on dense arrays of holes of less than $100 \mathrm{~nm}$ in diameter and spaced of a few tenths of $\mathrm{nm}$. Such systems produced from copolymer diblocks will be investigated thanks to the large number of NCs under the laser spot (a few units per window hole but a large density of holes. We believe that detecting a few units of Si-NCs is possible by improving the experimental set-up, which can be designed in order to maximize the brightness in detriment to resolution which is, however, not important in that case.

\section{References}

[1] J. Valenta, I. Pelant, J. Linnros, Appl. Phys. Lett. 81 (2002) 1396.

[2] R.J. Walters, et al., Appl. Phys. Lett. 85 (2004) 2622.

[3] P.W. Li, et al., Appl. Phys. Lett. 85 (2004) 1532 and refs therein.

[4] C. Bonafos, et al., Solid State Electron. 49 (2005) 1734.

[5] M. Shalchian, et al., Appl. Phys. Lett. 86 (2005) 163111.

[6] M. Carrada, et al., Appl. Phys. Lett. 87 (2005) 251911.

[7] M.V. Wolkin, et al., Phys. Rev. Lett. 82 (1999) 197. 